\title{
Sarcolipin expression in human skeletal muscle: Influence of energy balance and exercise
}

\author{
David Morales-Alamo 1,2 \\ Marcos Martin-Rincon ${ }^{1,2}$ (I) \\ Jose A. L. Calbet ${ }^{1,2,6}$ (i)
}

\author{
Miriam Martinez-Canton ${ }^{2}$
}

| Pedro de Pablos-Velasco ${ }^{2,4}$

Miriam Gelabert-Rebato ${ }^{1,2,3}$

| Hans-Christer Holmberg ${ }^{5}$ |

\author{
${ }^{1}$ Department of Physical Education, \\ University of Las Palmas de Gran Canaria, \\ Campus Universitario de Tafira s/n, Las \\ Palmas de Gran Canaria, Spain \\ ${ }^{2}$ IUIBS, Instituto de Investigaciones \\ Biomédicas y Sanitarias de Las Palmas de \\ Gran Canaria, Canary Islands, Spain \\ ${ }^{3}$ Nektium Pharma, Las Palmas de Gran \\ Canaria, Spain \\ ${ }^{4}$ Service of Endocrinology and Nutrition, \\ Hospital Universitario de Gran Canaria \\ Doctor Negrín, Las Palmas de Gran \\ Canaria, Spain \\ ${ }^{5}$ Department of Health Sciences, Swedish \\ Winter Sports Research Centre, Mid \\ Sweden University, Östersund, Sweden \\ ${ }^{6}$ Department of Physical Performance, \\ Norwegian School of Sport Sciences, Oslo, \\ Norway
}

\section{Correspondence}

Jose A. L. Calbet, Departamento de Educación Física, Campus Universitario de Tafira, 35017 Las Palmas de Gran Canaria, Canary Islands, Spain.

Email: lopezcalbet@gmail.com

\section{Funding information}

Instituto de Salud Carlos III, Grant/ Award Number: PI14/01509; Universidad de Las Palmas de Gran Canaria, Grant/ Award Number: ULPGC 2015/05; Agencia Canaria de Investigación Innovación y Sociedad de la Información, Grant/Award Number: ProID2017010106
Sarcolipin (SLN) is a SERCA uncoupling protein associated with exercise performance and lower adiposity in mice. To determine SLN protein expression in human skeletal muscle and its relationship with adiposity, resting energy expenditure (REE), and performance, SLN was assessed by Western blot in 199 biopsies from two previous studies. In one study, 15 overweight volunteers underwent a pretest followed by 4 days of caloric restriction and exercise (45-minute one-arm cranking +8 -hour walking), and 3 days on a control diet. Muscle biopsies were obtained from the trained and non-exercised deltoid, and vastus lateralis $(V L)$. In another study, 16 men performed seven sessions of 4-6 $\times 30$-sec all-out sprints on the cycle ergometer with both limbs, and their $V L$ and triceps brachii biopsied pre- and post-training. SLN expression was twofold and $44 \%$ higher in the $V L$ than in the deltoids and triceps brachii, respectively. SLN was associated with neither adiposity nor REE, and was not altered by a severe energy deficit ( $5500 \mathrm{kcal} / \mathrm{day})$. SLN and cortisol changes after the energy deficit were correlated $(r=.38, P=.039)$. SLN was not altered by low-intensity exercise in the overweight subjects, whereas it was reduced after sprint training in the other group. The changes in SLN with sprint training were inversely associated with the changes in gross efficiency $(r=-.59, P=.016)$. No association was observed between aerobic or anaerobic performance and SLN expression. In conclusion, sarcolipin appears to play no role in regulating the fat mass of men. Sprint training reduces sarcolipin expression, which may improve muscle efficiency.

\section{K E Y W O R D S}

exercise, obesity, resting energy expenditure, sarcolipin, sprint training 


\section{1 | INTRODUCTION}

Sarcolipin (SLN) is a 31-amino-acid protein expressed only in striated and cardiac muscle, ${ }^{1}$ which regulates muscle thermogenesis. ${ }^{2}$ SLN expression increases with overloading ${ }^{3}$ and is associated with enhanced exercise performance in mice. ${ }^{4}$ It remains unknown whether, in humans, the expression of SLN is influenced by loading and energy balance. Although it has been suggested that enhancing SLN activity could be an effective mechanism to increase energy expenditure and control weight gain, ${ }^{5}$ the role that SLN may play in the regulation of fat mass is unknown in humans. ${ }^{6}$

Skeletal muscle accounts for $15 \%-17 \%$ of resting metabolic rate (RMR). ${ }^{7}$ The majority of the energy expended by resting skeletal muscles is used to maintain the resting membrane potential and cytosolic $\left[\mathrm{Ca}^{2+}\right]$, through the action of the $\mathrm{Na}^{+}-\mathrm{K}^{+}$ATPase and the sarcoplasmic reticulum $\mathrm{Ca}^{2+}$-ATPase (SERCA). ${ }^{8}$ In mice, skeletal muscles' ATP consumption by the SERCA accounts for $40 \%-50 \%$ of the muscles' RMR or $12 \%-15 \%$ of the whole-body $\mathrm{VO}_{2} .{ }^{9}$ In optimal conditions, SERCA harnesses the energy released by one ATP phosphate bond to translocate two $\mathrm{Ca}^{2+}$ ions into the sarcoplasmic reticulum. ${ }^{10}$ The thermodynamic efficiency of SERCA is reduced by SLN binding, which decreases the net $\left(V_{\max }\right)$ of $\mathrm{Ca}^{2+}$ uptake and uncouples $\mathrm{Ca}^{2+}$ transport from ATP hydrolysis. ${ }^{11}$ Mice exposed to cold can upregulate SLN expression to lower the SERCA $\mathrm{Ca}^{2+} / \mathrm{ATP}$ coupling ratio, resulting in increased ATP hydrolysis and heat production through the futile cycling of SERCA. ${ }^{12}$

Mice lacking sarcolipin $\left(\mathrm{SLN}^{--}\right)$become more obese than the wild-type (WT) littermates when on a high-fat diet (HFD), ${ }^{2}$ while mice overexpressing $\mathrm{SLN}\left(\mathrm{SLN}^{\mathrm{OE}}\right)$ are resistant against diet-induced obesity. ${ }^{13}$ Compared to WT, $\mathrm{SLN}^{\mathrm{OE}}$ mice have higher daily energy expenditure $(+27 \%)$, which has been attributed to their increased skeletal muscle $\mathrm{VO}_{2}$ both at rest and during electrical stimulation. ${ }^{13}$ These mouse studies support a role for SLN in the regulation of fat mass such that higher levels of SLN expression could protect against obesity and vice versa. In contrast, experiments using human skeletal muscle cells (HSkMC) from seven lean and seven extremely obese women, which were differentiated into primary myocytes, reported a 3.5 -fold higher SLN protein in the myocytes derived from HSkMC originating from obese women. ${ }^{14}$ Nevertheless, Paran et al ${ }^{14}$ did not report skeletal muscle SLN protein expression nor RMR data. Thus, it remains unknown whether SLN in human skeletal muscle is associated with the degree of obesity and the magnitude of the RMR. Moreover, no study in humans has determined whether SLN protein expression is regulated in response to energy balance and exercise.

In rodents, SLN protein expression is relatively high in fetal and neonatal skeletal muscles but gradually becomes restricted to slow-twitch oxidative muscle fibers. ${ }^{1}$ Compared to rodents, SLN mRNA expression levels are several folds higher in slow- and fast-twitch muscles of larger mammals. ${ }^{15}$ Comparative analyses of SLN protein expression levels in different human skeletal muscles are lacking. Moreover, functional overloading of plantaris muscle is associated with increased SLN mRNA ${ }^{3}$ and protein expression ${ }^{16}$ in mice suggesting that chronic overload could stimulate SLN expression. It remains unknown how SLN expression responds to substantially different loading patterns in different human skeletal muscles. This knowledge could help in designing training programs aiming to either enhance or reduce SLN expression in specific muscle groups. A higher level of SLN in skeletal muscle may facilitate energy expenditure and could be of interest for patients with obesity. On the other hand, reducing SLN expression levels could enhance muscle efficiency and improve endurance.

Thus, the primary aim of this study was to determine whether SLN protein expression is increased in skeletal muscles of overweight men submitted to high (vastus lateralis) and low (deltoid) daily loading and associated with the percentage of body fat. Secondary aims were as follows: (a) to find out whether SLN expression in skeletal muscle is associated with myosin heavy chain composition; (b) to ascertain whether SLN protein expression is modified in human skeletal muscle in response to a large negative energy balance to reduce RMR; and (c) to determine whether SLN protein expression is altered by low- and high-intensity exercise.

To achieve these aims, we used muscle biopsies from two previous studies. ${ }^{17-20}$ The "Östersund walking study" was designed to induce a severe energy deficit of $5500 \mathrm{kcal} /$ day, for 4 days, by energy restriction $(3.2 \mathrm{kcal} / \mathrm{kg}$ body weight/day) and prolonged exercise (8-hour walking). This energy deficit was chosen to elicit a 2-3 kg reduction in body fat mass, as shown by dual-energy X-ray absorptiometry. ${ }^{18}$ Before walking, the subjects exercised one arm for 45 minutes at low-intensity, while the other arm did not exercise and served as a control. This offered the opportunity to examine the impact of a severe energy deficit with reduction of fat mass on SLN expression in different skeletal muscles from the same person submitted to no exercise and a small or large amount of low-intensity exercise. The subjects participating in the "Östersund walking study" were overweight, allowing to test whether SLN protein expression is related to the degree of adiposity. To study the effect of high-intensity exercise training on SLN expression in human skeletal muscle, we used biopsies obtained from volunteers of the same population that participated in a sprint training program. ${ }^{19,20}$

Based on a greater SLN expression in the fast muscle fibers of larger mammals, ${ }^{1}$ we hypothesized that the protein expression of SLN will be associated with a greater MHC type II composition in human skeletal muscles and that a severe energy deficit would induce a reduction of SLN expression, as a mechanism to lower RMR. We also hypothesized 
that large volumes of low-intensity exercise would reduce SLN expression, while high-intensity exercise training would increase the expression of SLN as a mechanism to improve muscle energetics and reduce fatigue, as observed in $\mathrm{SLN}^{\mathrm{OE}}$ mice. $^{4}$

\section{2 | METHODS}

\section{1 | Participants}

The characteristics of the two groups studied, experimental design, and general procedures have been reported previously. Fifteen physically active overweight men participated in the "Östersund walking study"17,18,21 (hereafter called "overweight walking group"), and sixteen men from the same population, fourteen of them with a body mass index (BMI) $<25 \mathrm{~kg} / \mathrm{m}^{2}$ and two overweight participated in a 2-week sprint training study ${ }^{19,20}$ on the cycle ergometer (Table 1). The fourteen subjects with a BMI $<25$ were considered as "lean" and were compared to the overweight subjects of the Östersund walking study. The inclusion criteria for participants in the "Östersund walking study" have been previously reported. ${ }^{18}$ Briefly, the inclusion criteria were as follows: age between 18 and 55 years, stable body weight for the preceding three months before the start of the study, BMI of $\geq 25 \mathrm{~kg} / \mathrm{m}^{2}$, waist circumference of $>102 \mathrm{~cm}$, and body fat percentage between $20 \%$ and $40 \%$. Exclusion criteria were as follows: orthopedic medical contraindications for prolonged walking, smoking, or chronic diseases. The subjects that participated in the sprint training study were physically active non-smokers, exercising recreationally two or three times a week. After being informed of potential risks and benefits, all subjects provided their written consent to participate. Ethical approval was obtained from the Regional Ethical Review Board of Umeå University (Umeå, Sweden; 2010/236-31 and 2014/91-31).

\section{2 | Experimental protocol (overweight walking group)}

The protocol for the overweight group consisted of a pretest phase (PRE), caloric restriction combined with exercise for 4 days (CRE), immediately followed by a control diet with reduced exercise for 3 days (CD; Figure 1A). During the PRE, and at the end of the CRE and CD phases, body composition was assessed (Lunar iDXA, GE Healthcare) ${ }^{22}$; $20 \mathrm{~mL}$ blood samples were drawn; resting metabolic rate was measured, ${ }^{23}$ and muscle biopsies were then taken from both deltoid muscles and the vastus lateralis of one leg (randomly assigned). All of these procedures were performed in the morning following a 12-hour overnight fast. ${ }^{18,21}$

\subsection{Experimental protocol (sprint training group)}

The protocol for the sprint training group consisted of an initial phase of pretests when blood samples and muscle biopsies were taken from the triceps brachii $(T B)$ and the ipsilateral vastus lateralis. This was followed by seven training sessions performed on the leg cycle ergometer or an ergometer modified for arm cycling in 2 weeks, which included repeated sprints performed with either first the arms followed (1-hour rest period) by the legs or vice versa, according to the randomization assignment (Figure 1B). Half of the subjects trained first with the arms, while the other half started first with the legs. For both arms and legs, the subjects were instructed to perform (4-6) repeated 30-second all-out sprints, separated by 4 minutes of recovery (at unloaded pedaling or cranking at $\sim 20 \mathrm{rpm}$ ). On the first and second days of training, each subject performed four sprints; on the third and fourth days, five sprints; and on the final 3 days, six sprints, for a total of 36 sprints with the arms and an equal number with the legs. Overall, subjects performed seven sprint training sessions over 2 weeks, and 48 hours later, the muscle biopsies and blood samples were repeated (Figure 1B). ${ }^{19}$

\subsection{Additional assessments}

The methods used to carry out the rest of the assessment are reported in full detail in previous publications. ${ }^{17-19}$ Briefly, body composition was assessed using dual-energy X-ray absorptiometry (Lunar iDXA, GE Healthcare), and peak oxygen uptake ( $\mathrm{VO}_{2}$ peak) (AMIS 2001 model C, Innovision A/S) was determined by an incremental exercise test to exhaustion on the cycle ergometer (SRM GmbH). In the overweight walking group, one-arm $\mathrm{VO}_{2}$ peak was determined using an arm cranking incremental exercise test to exhaustion performed with each arm separately using a similar ergometer. ${ }^{17}$ In the sprint training group, arm cranking $\mathrm{VO}_{2}$ peak was assessed using an incremental arm cranking test to exhaustion carried out with both arms. ${ }^{19}$ The $\mathrm{VO}_{2}$ data collected during the incremental exercise tests were averaged every 20 seconds, and the highest 20 -second average was taken as the $\mathrm{VO}_{2}$ peak, as recommended elsewhere. ${ }^{24}$

In the sprint training group, additional performance tests to measure peak $\left(W_{\text {peak }}\right)$ and mean $\left(W_{\text {mean }}\right)$ power output during 30-second sprint tests (Wingate tests) were performed. The Wingate tests were followed by 20 minutes of rest and, immediately after, by a 5 -minute time trial (TT) ${ }^{19}$

\section{5 | Resting metabolic rate}

In the overweight group, the resting metabolic rate (RMR) was assessed after a 12-hour overnight fast following at least 
TABLE 1 Physical characteristics, body composition, and $\mathrm{VO}_{2 \max }$

\begin{tabular}{|c|c|c|c|c|}
\hline & \multicolumn{2}{|c|}{$\begin{array}{l}\text { Overweight subjects at PRE } \\
(n=15)\end{array}$} & \multicolumn{2}{|c|}{ Sprint training study $(\mathrm{n}=16)$} \\
\hline & Pre-intervention & Post-CD & Pre-training & Post-training \\
\hline Age (y) & $41 \pm 8.1$ & & $23.9 \pm 3.7 * *$ & \\
\hline Height $(\mathrm{cm})$ & $181 \pm 5$ & & $184 \pm 7$ & \\
\hline Weight (kg) & $99.1 \pm 13.2$ & $95.3 \pm 12.6^{*}$ & $77.8 \pm 6.9^{a, * *}$ & $77.7 \pm 6.8^{\mathrm{a}, * *}$ \\
\hline BMI $\left(\mathrm{kg} / \mathrm{m}^{2}\right)$ & $30.4 \pm 3.7$ & $29.2 \pm 3.5^{*}$ & $23.7 \pm 2.8^{* *}$ & $23.7 \pm 2.8^{* *}$ \\
\hline Lean mass $(\mathrm{kg})$ & $64.3 \pm 4.9$ & $63.3 \pm 4.5^{*}$ & $60.9 \pm 5.8^{\mathrm{a}, * *}$ & $61.1 \pm 5.8^{\mathrm{a}, * *}$ \\
\hline $\begin{array}{l}\text { Legs lean mass } \\
(\mathrm{kg})\end{array}$ & $23.0 \pm 2.2$ & $22.6 \pm 2.1^{*}$ & $21.0 \pm 2.0 * *$ & $21.0 \pm 2.1 * *$ \\
\hline $\begin{array}{l}\text { Arms lean } \\
\text { mass (kg) }\end{array}$ & $8.4 \pm 0.8$ & $8.1 \pm 0.7^{*}$ & $8.3 \pm 1.1$ & $8.3 \pm 1.0$ \\
\hline Fat mass (kg) & $31.5 \pm 8.8$ & $28.6 \pm 8.5^{*}$ & $13.5 \pm 4.2^{\mathrm{a}, * *}$ & $13.2 \pm 3.9^{\mathrm{a}, * *}$ \\
\hline Body fat $(\%)$ & $31.2 \pm 4.6$ & $29.5 \pm 4.9^{*}$ & $18.1 \pm 4.9^{\mathrm{a}, * *}$ & $17.8 \pm 4.7^{\mathrm{a}, * *}$ \\
\hline $\begin{array}{l}\mathrm{VO}_{2 \max }(\mathrm{L} / \\
\min )\end{array}$ & $3.84 \pm 0.33$ & $3.61 \pm 0.42$ & $3.79 \pm 0.44$ & $4.08 \pm 0.45^{*}$ \\
\hline
\end{tabular}

Note: Values are mean $\pm \mathrm{SD}$.

In the overweight group, 10 subjects had a BMI $25 \geq$ and $<30$, and the other five had a BMI $>30$. Post-CD: after $3 \mathrm{~d}$ on a control diet with reduced exercise.

${ }^{\mathrm{a}}(\mathrm{n}=15)$

$* P<.01$ compared to the pre-training value.

$* * P<.05$ compared to the overweight group.
10 minutes of resting in the supine position using the same

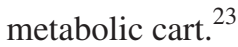

\section{6 | Muscle biopsies, extraction of total protein, Western blotting, and myosin heavy chain composition analysis}

Muscle biopsies were taken by the same medical doctor using similar procedures in both studies. ${ }^{19,21}$ Briefly, $30 \mathrm{mg}$ of muscle was homogenized in urea lysis buffer and $15 \mu \mathrm{g}$ of protein extract loaded onto each gel lane, and run together with four control samples. The gels were incubated overnight at $4^{\circ} \mathrm{C}$. The same control extract was loaded in all gels to assure comparability between gels. ${ }^{25}$ Proteins were transferred to Immun-Blot PVDF Membranes and subsequently stained with Reactive Brown $10^{26}$ to control for differences in loading and transfer efficiency, and the bands captured with the ChemiDocTM Touch Imaging System and quantified with the Image Lab 5.2.1 software. The membranes were blocked for 1 hour in $4 \%$ bovine serum albumin in Tris-buffered saline containing
0.1\% Tween-20 (TBS-T; BSA-blocking buffer) and incubated overnight at $4{ }^{\circ} \mathrm{C}$ with the anti-sarcolipin antibody (catalog no. ABT13, EMD-Millipore) diluted 1:2000 in 4\% BSA-blocking buffer. The protein bands were revealed by incubation with an HRP-conjugated anti-rabbit antibody (diluted 1:5,000 in Blotto blocking buffer) and visualized using Clarity ${ }^{\mathrm{TM}}$ Western ECL Substrate (Bio-Rad Laboratories) using a ChemiDoc ${ }^{\text {TM }}$ Touch Imaging System (Bio-Rad Laboratories). Finally, the bands were quantified with the Image $\mathrm{Lab}^{\odot}$ software 5.2.1 (Bio-Rad Laboratories). Since loading was homogeneous in all membranes, the native band densities (without corrections for loading) were directly used, to avoid unnecessarily increasing the variability. ${ }^{25}$ Myosin heavy chain composition was determined as previously reported. ${ }^{27}$

\section{7 | Biochemical and hormonal analyses}

A detailed description of hormonal and plasma amino acid analysis is reported in previous publications. ${ }^{17,21}$ Briefly, serum insulin was quantified by an electrochemiluminescence

F I G U R E 1 A, Schematic illustration of the experimental protocol for the overweight walking group. PRE: pretest; CRE: four days of caloric restriction (320 kcal/day) and exercise (45 min of single-arm cranking and $8 \mathrm{~h}$ of walking each day); CD: three days on a diet isoenergetic (compared with that observed during the PRE phase) with reduced exercise ( $<10000$ steps per day). B, Experimental protocol for the sprint training group. All exercise bouts during testing and training were 30-s all-out sprints performed employing both leg pedaling (left) and arm cranking. The values between brackets indicate the number of 30-s sprints per training session. DXA: dual-energy X-ray absorptiometry; IPAQ: international physical activity questionnaire 
(A)

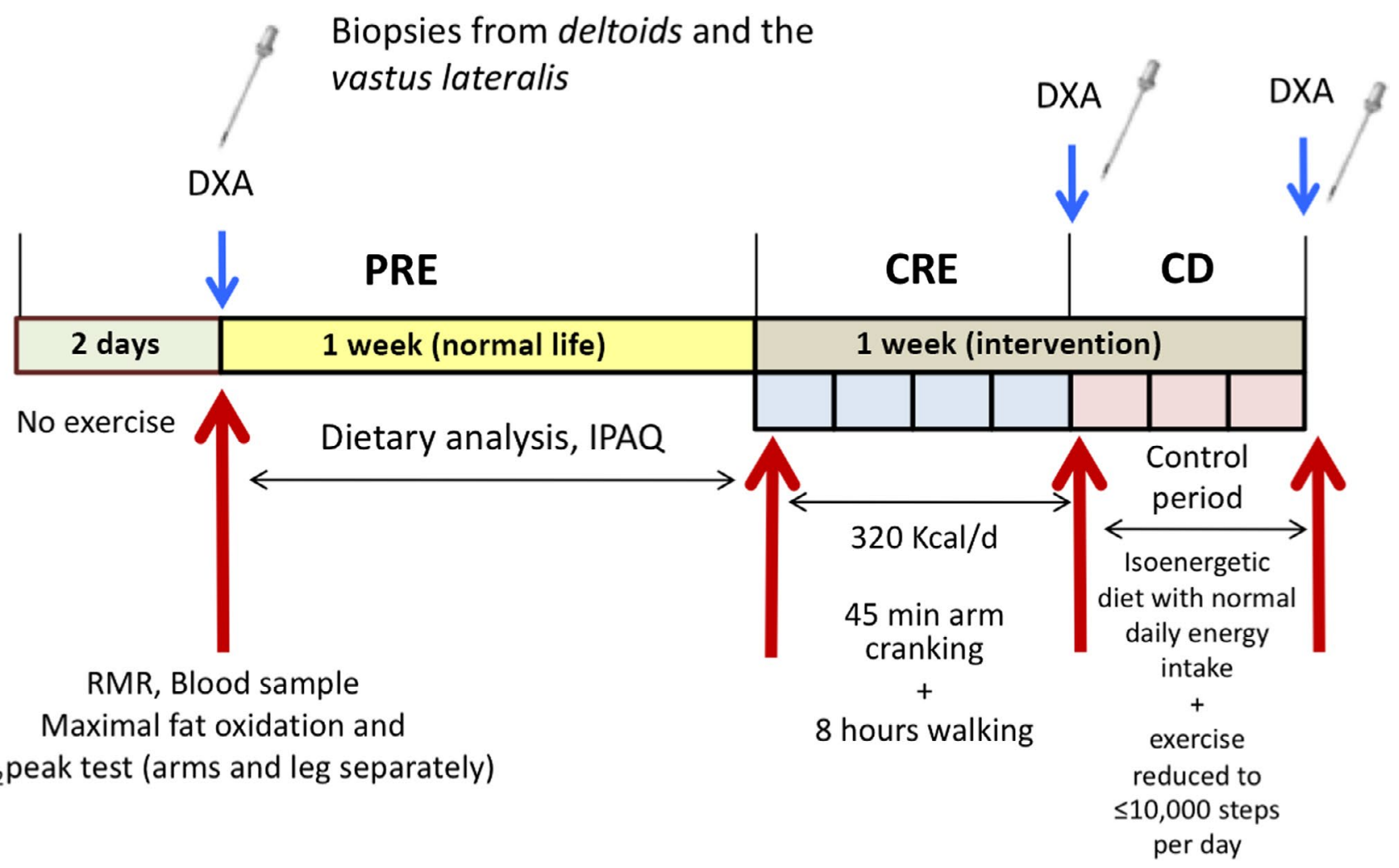

(B)

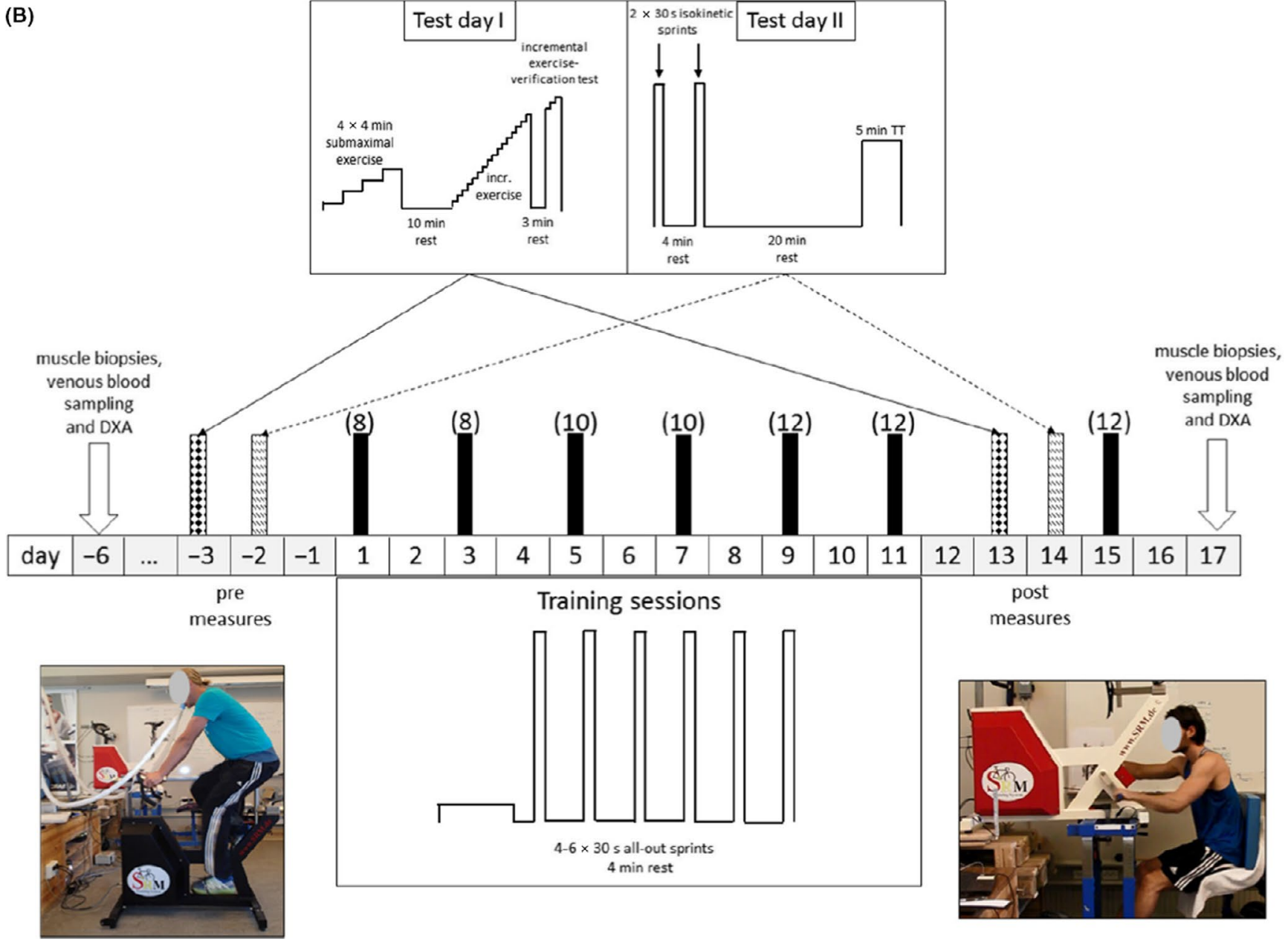


immunoassay (Modular Analytics Analyzer E170, Roche Diagnostics SL, Barcelona, Spain), at a sensitivity of $0.2 \mu \mathrm{U} /$ $\mathrm{mL}$ (intra- and inter-assay coefficients of variation $(\mathrm{CV})$ of 2.0 and $2.6 \%$, respectively). Cortisol and total testosterone were measured with chemiluminescence enzyme immunoassays (Immulite 2000 Cortisol, Ref. L2KCO2, Immulite 2000 Total Testosterone, Ref. L2KTW2; Siemens) at a sensitivity of 5.5 and $0.5 \mathrm{nmol} / \mathrm{L}$ and intra- and inter-assay $\mathrm{CV}$ of $6.2 \%$ and $7.3 \%$, and $8.2 \%$ and $9.1 \%$, respectively. Free testosterone was determined by a radioimmunoassay (Coat-A-Count Free Testosterone, Ref. TKTF1; Siemens) with a sensitivity of $0.5 \mathrm{pmol} / \mathrm{L}$ and intra- and inter-assay $\mathrm{CV}$ of less than $8 \%$. Serum leptin was determined by the enzyme-linked immunosorbent assay (Linco Research \#EZHL-80SK) with a sensitivity of $0.05 \mathrm{ng} / \mathrm{mL}$ and intra- and inter-assay CV of 3.8\% and $4.4 \%$, respectively.

Following automated precolumn derivatization of plasma amino acids with o-phthalaldehyde, the resulting derivatives were separated by reversed-phase HPLC (on a 5-m Resolve C18 column; Waters) and quantified by fluorescence detection.

\section{8 $\quad$ Statistical analyses}

Descriptive values are presented as means \pm standard deviations (SD). The statistical analysis was based on repeatedmeasures ANOVAs. Pairwise comparisons ( $t$ tests) at specific time-points were adjusted for multiple comparisons with the Holm-Bonferroni procedure. Comparisons between the overweight group (PRE samples) and sprint training groups (PRE training samples) were performed using the unpaired $t$ test. Associations between variables were evaluated using linear regression. All of these analyses were performed in the SPSS v.21 software for Windows (SPSS Inc).

\section{$3 \mid$ RESULTS}

The general effects of the interventions are reported with more detail in previous publications. ${ }^{17-21,28}$ Briefly, in the overweight walking group, the combination of caloric restriction with prolonged exercise elicited an energy deficit of $5500 \mathrm{kcal} /$ day. ${ }^{18}$ Consequently, from PRE to CRE and CD, total body mass was reduced by an average of 4.9 and $3.9 \mathrm{~kg}$ and fat mass by 2.1 and $2.8 \mathrm{~kg}$, respectively $(P<.001){ }^{18}$ This energy deficit was associated with reductions in serum concentrations of glucose, insulin, testosterone, free testosterone, and leptin, whereas serum cortisol concentration increased. ${ }^{17,18}$ The resting metabolic rate was not significantly altered by the intervention. The vastus lateralis exhibited a higher proportion of MHC I than the deltoids (52.2 \pm 14.4 and $41.5 \pm 5.5 \%$, respectively, $P=.005) .{ }^{21}$
No significant changes in body composition were observed in the sprint training group. As previously reported, this training elicited improvements in $\mathrm{VO}_{2}$ peak, Wingate test peak and mean power outputs, time trial performance, and arm cranking gross efficiency ( $9 \%)$. Delta efficiency was $42 \%$ greater for the leg than arm exercise and not significantly altered by training $(P=.43) .{ }^{19}$ The $V L$ exhibited higher MHC I percentage than the triceps brachii $(44.4 \pm 10.7$ and $26.4 \pm 9.0 \%$, respectively, $P<.001) .{ }^{19}$

\section{1 | Sarcolipin protein expression in human skeletal muscle and adiposity}

The protein expression of sarcolipin in human skeletal muscle presents marked differences between both individual muscles and subjects. The vastus lateralis expressed twofold more SLN than the deltoid muscles in the overweight group $(P<.001$; Figure $2 \mathrm{~A})$ and $44 \%$ more than the triceps brachii in the sprint training group $(P=.002$; Figure $2 \mathrm{~B})$. There was a linear relationship between the level of SLN expression in the arm (mean of both deltoids) and vastus lateralis in the overweight group $(r=.76, P<.001)$. A similar relationship was confirmed between the triceps brachii and the vastus lateralis in the lean subjects $(r=.95$, $P<.001)$. The deltoids and the triceps brachii of the overweight and lean subjects had similar SLN protein expression levels $(P=.59$; Figure $2 \mathrm{~B})$. Likewise, the vastus lateralis expressed similar amounts of SLN in both groups $(P=.25$; Figure 2B).

At baseline, vastus lateralis SLN protein expression was linearly related to the percentage of MHC II in the overweight group ( $r=.65, P=.009, \mathrm{n}=15$; Figure 3$)$, but not in the lean group $(r=-0.25, P=.38, \mathrm{n}=14)$. No association between SLN protein expression and the percentage of MHC II was observed for the arm muscles in either group $(r=.44$, $P=.10, \mathrm{n}=15$ and $r=.17, P=.52, \mathrm{n}=14$, for the averaged of both arms in the overweight and one arm in the lean groups, respectively).

No significant associations were observed between SLN protein expression and the percentage of wholebody or trunk fat (data not shown) neither in overweight (Figure 4A,C,E) nor the lean group (data not shown). Similar results were observed when both groups were combined, that is, no association was observed between the percentage of whole-body fat and SLN protein expression $(r=.21, P=.42)$. When both groups were combined, there was no association between SLN protein expression and RMR (in $\mathrm{kcal} / \mathrm{min} / \mathrm{kg}$ body mass) at PRE (Figure $4 \mathrm{~B}, \mathrm{D}, \mathrm{F})$. The changes in SLN expression were not associated with the changes in RMR (data not shown) in the overweight group. 
(A)

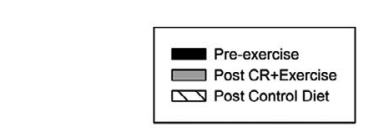

Time effect $P=0.76$

Extremity effect $P<0.001$

Time by supplementation $P=0.85$

Extremity by time $P=0.27$

Extremity by supplementation $P=0.68$

Extremity by time by supplementation $P=0.30$

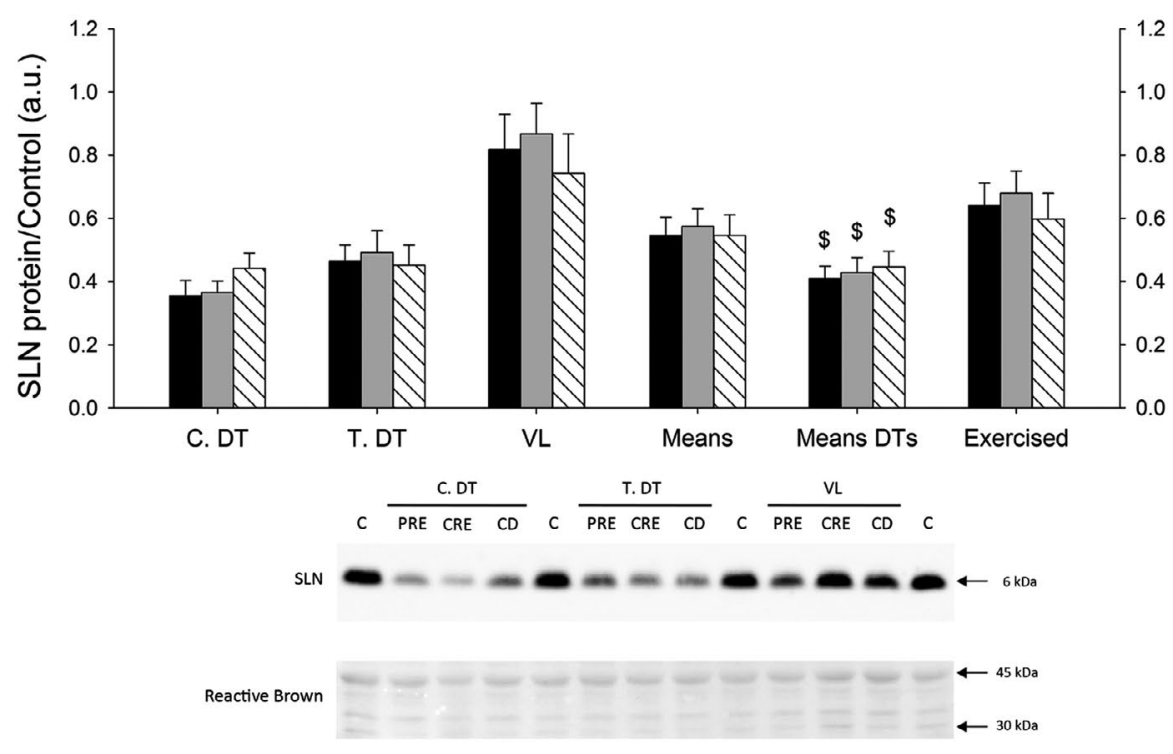

$\$$ Compared to Legs $(P<0.05)$

FIGURE 2 Sarcolipin protein expression levels and representative blots. A, Overweight walking group $(\mathrm{n}=15)$. SLN: sarcolipin; C. DT: control deltoid muscle (non-exercised); T. DT: trained deltoid muscle; VL: vastus lateralis muscle; means: $(\mathrm{C}$. DT + T. DT + VL)/3; means DTs: (C. DT + T. DT)/2; exercised: (T. $\mathrm{DT}+\mathrm{VL}) / 2$. B, Sprint training group $(\mathrm{n}=16)$, both before (black bars) and after (gray bars) training. The mean values of both muscles in the right part of the figure represent the mean values of both muscles before (white bar) and after (hatched bar) training. Triceps B.: triceps brachii; Vastus L: vastus lateralis. Values are normalized to the mean value of control muscle extract and presented as arbitrary units (a.u.). The error bars represent the standard error $(n=15)$. $* P<.05$ compared to before training. PRE; $\$ P<.05$ compared to the legs

(B)

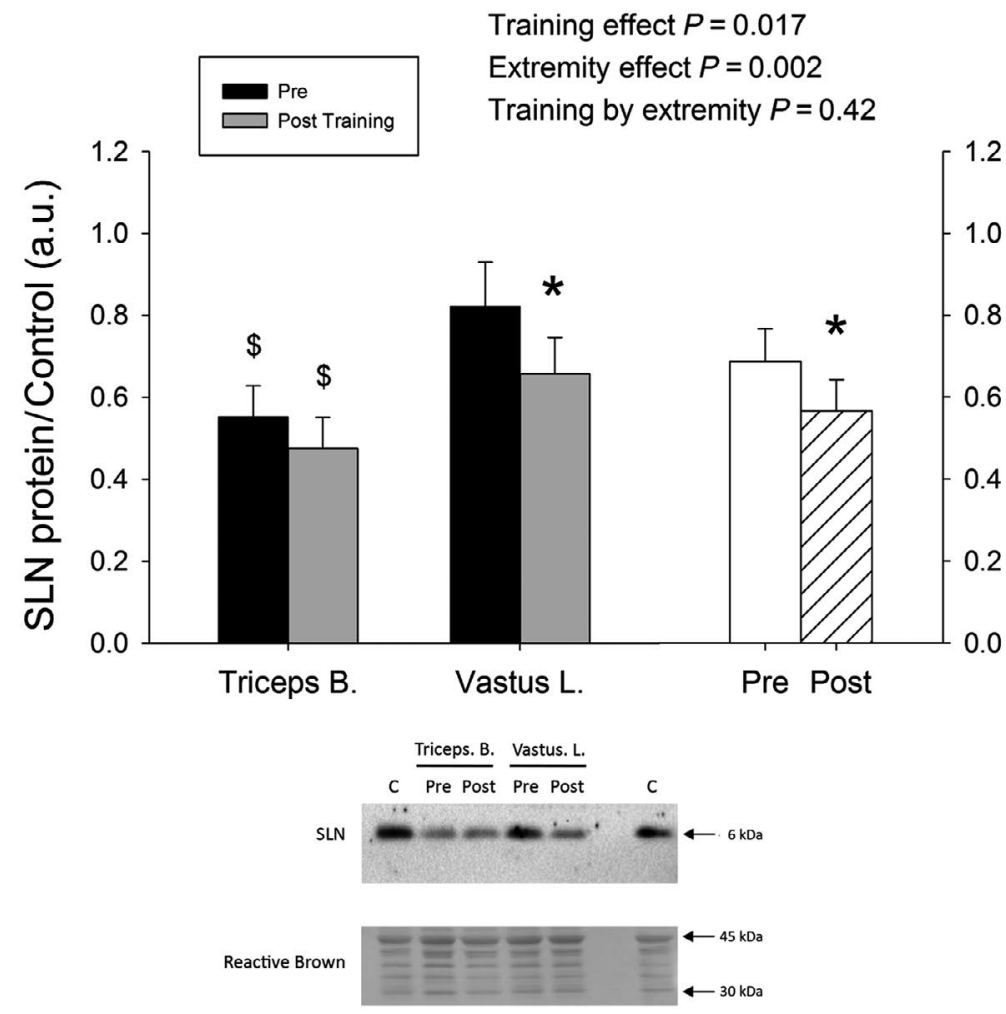

\section{2 | Sarcolipin expression and exercise}

In the overweight group, SLN protein expression was not significantly altered by exercise, caloric restriction, or the type of supplement administered during CRE (Figure 2A). SLN protein expression was reduced by $18 \%$ after sprint training

$(P=.017)$, mostly due to a $20 \%$ drop in the vastus lateralis $(P=.027$; Figure 2B).

Despite the lower SLN expression in the arm than leg muscles, both delta and gross efficiency were lower for arm cranking than leg pedaling ${ }^{19}$ in the sprint training group. Nevertheless, there was a trend for a negative association 


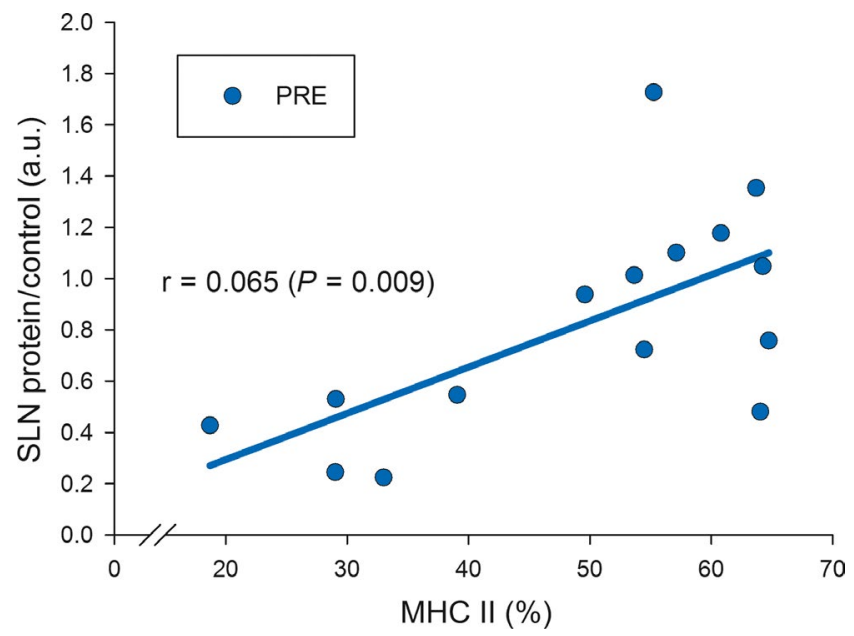

F I G U RE 3 Relationship between sarcolipin (SLN) protein expression in the vastus lateralis and myosin heavy chain type II composition (MHC II) in the overweight walking group before the intervention. Sarcolipin values are normalized to the mean value of control muscle extract and presented as arbitrary units (a.u.). $\mathrm{n}=15$

between SLN protein expression in the legs (average of PRE and post-training) and the mean delta efficiency during pedaling (logarithm of the average of PRE and post-training; $r=-.48, P=.058)$. The improvement in gross efficiency during arm cranking was not associated with the changes observed in SLN $(r=-.28, P=.29)$. However, the small changes in leg pedaling gross efficiency were inversely and linearly related to the changes in SLN expression $(r=-.59$, $P=.016)$. No relationship was observed between SLN protein expression and performance in either group.

Neither time trial nor Wingate test performance during arm cranking and leg pedaling was related to the respective SLN expression in the arm and leg muscles. The improvements in time trial and Wingate test performance after training were not associated with the changes in SLN expression (data not shown).

\section{3 | Relationships between sarcolipin protein expression, metabolites, and hormones in the overweight group}

The changes in SLN protein expression (the mean of the two deltoids averaged with the vastus lateralis) from PRE to CRE and from PRE to CD were directly associated with the corresponding changes in cortisol $(r=.38, P=.039$; Figure 5). No significant associations were observed between the baseline levels of SLN protein expression and the serum concentrations of cortisol, total testosterone, free testosterone, leptin, insulin, HOMA, essential amino acids, branched amino acids, thyroid hormones, or cortisol/testosterone ratio.

\section{DISCUSSION}

This study shows that the protein sarcolipin is expressed in human skeletal muscle with considerable differences between subjects and upper and lower extremities, as reflected by the analysis of 199 muscle biopsies. The vastus lateralis expresses twice the amount of SLN observed in the deltoid and about $44 \%$ more than the triceps brachii. In partial agreement with our hypothesis, SLN expression was positively associated with the percentage of MCH II, but exclusively in the vastus lateralis of the overweight men. In contrast to our hypothesis, SLN expression was not associated with adiposity nor resting energy expenditure in overweight men, and therefore, similar levels of SLN expression were observed in overweight and lean men from the same population. Our results reveal an association between sprint training-induced changes in SLN expression and energy efficiency, which fits with a putative influence of SLN on exercise energy expenditure. We have also shown that SLN protein levels in human skeletal muscle are not altered by a severe energy deficit $(\sim 5500 \mathrm{kcal} /$ day for 4 days) that lowers fat mass by $2.8 \mathrm{~kg}$. Importantly, this investigation has demonstrated that while SLN protein expression does not seem to change with lowintensity exercise, it is reduced after just seven sessions of sprint training. Altogether, these results indicate that in contrast to previous postulates based on mouse data, SLN protein levels in skeletal muscles are not associated with resting energy expenditure or adiposity, in lean nor overweight humans. Finally, the present findings are compatible with a role of cortisol in the regulation of SLN protein levels in human skeletal muscle.

\section{1 | Sarcolipin protein is more abundantly expressed in leg than arm skeletal muscles in humans}

In agreement with our findings, the first data published on mRNA SLN expression in skeletal muscle showed SLN to be highly expressed in fast-twitch skeletal muscles and to a lesser extent in slow-twitch muscles and myocardium in rabbits and human beings. ${ }^{15}$ Protein levels of SLN were assessed in various muscle tissues of mouse, rat, rabbit, and dog by Western blot analysis for the first time in $2007 .{ }^{1}$ In rodents, the SLN protein was only found in soleus muscle (predominantly composed by slow-twitch fibers in mice) and diaphragm, while it was not detected in Quadriceps (predominantly composed by fast-twitch fibers in mice). ${ }^{1}$ Nonetheless, in larger mammals, ${ }^{1}$ SLN protein levels were detected in both slow- and fast-twitch skeletal muscles, but more abundantly in the latter. Here, we have observed a statistically significant linear relationship between SLN protein expression and MCH II proportion in the vastus lateralis but 

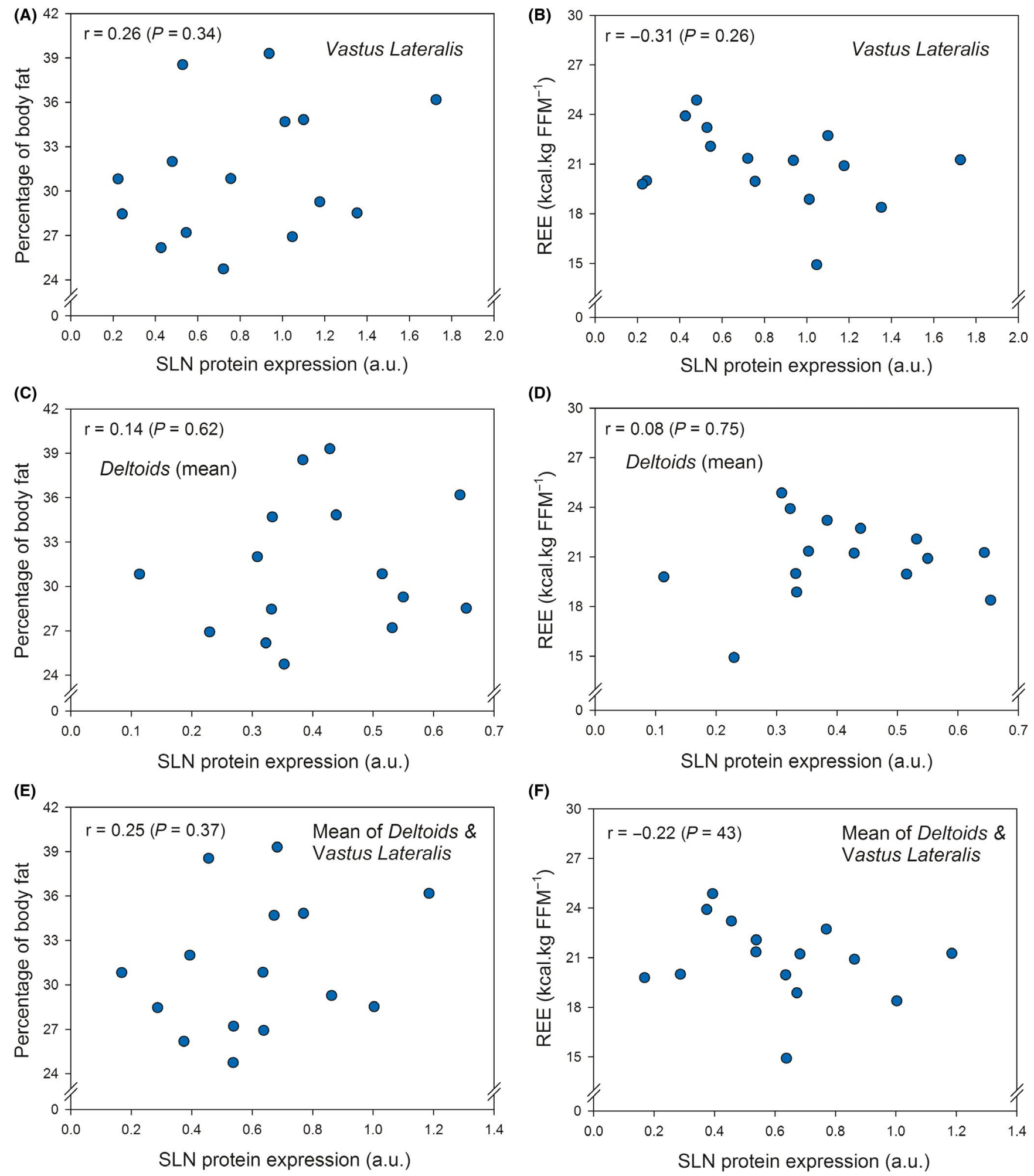

F I G U RE 4 Sarcolipin (SLN) protein expression, percentage of body fat, and resting energy expenditure (REE) in the overweight walking group before the intervention. A, Relationship between SLN protein expression in the vastus lateralis and the percentage of whole-body fat $(\mathrm{n}=15)$. B, Relationship between SLN protein expression in the vastus lateralis and resting energy expenditure $(\mathrm{n}=15)$. C, Relationship between SLN protein expression in the arms (mean of both deltoid muscles) and the percentage of whole-body fat $(n=15)$. D, Relationship between SLN protein expression in the arms (mean of both deltoid muscles) and resting energy expenditure $(\mathrm{n}=15)$. E, Relationship between the overall mean SLN protein expression (mean of both arms averaged with the vastus lateralis value) and the percentage of whole-body fat ( $\mathrm{n}=15$ ). $\mathrm{F}$, Relationship between the overall mean SLN protein expression (mean of both arms averaged with the vastus lateralis value) and resting energy expenditure. FFM: fat-free mass $(n=15)$. Sarcolipin values are normalized to the mean value of control muscle extract and presented as arbitrary units (a.u.) 


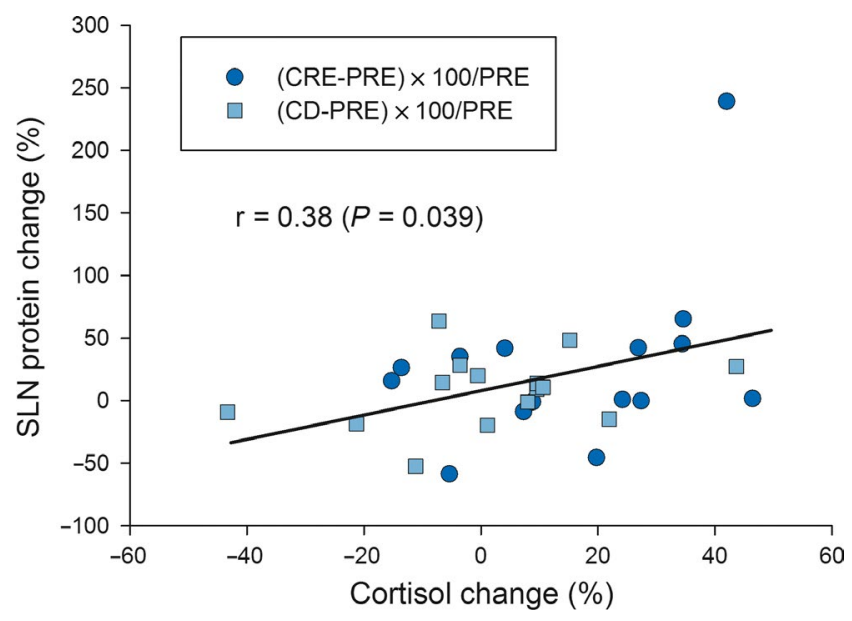

F I G URE 5 Changes in sarcolipin (SLN) protein expression and serum cortisol. Percentage change in skeletal muscle sarcolipin protein expression (mean of the two deltoid muscles averaged with the vastus lateralis) plotted against the corresponding change in serum cortisol concentration. PRE: pretest; CRE: four days of caloric restriction (320 kcal/day) and exercise (45 min of single-arm cranking and $8 \mathrm{~h}$ of walking each day); $\mathrm{CD}$ : three days on a diet isoenergetic (compared with that observed during the PRE phase) with reduced exercise $(<10000$ steps per day)

not in the deltoid nor triceps brachii muscles, although this finding was observed in a relatively small sample $(n=15)$. The reason why this association was only observed in the vastus lateralis of the overweight subjects is not due to differences in MHC II composition since both groups had similar values. Although both groups had comparable height and lean mass in the upper extremities, the overweight subjects had $\sim 10 \%$ greater lean mass in the lower extremities reflecting an increased mechanical loading due to their excessive body mass. Even though functional overloading has been associated with increased SLN expression in mice, ${ }^{3,16}$ no significant differences in vastus lateralis SLN protein expression were observed between the overweight and lean subjects. A potential explanation for this finding could be that much larger overloading may be required to increase SLN expression in humans.

\section{2 | Caloric restriction does not alter sarcolipin protein expression}

Caloric restriction and weight loss are associated with decreases in RMR and adaptive thermogenesis. ${ }^{29,30}$ Adaptive thermogenesis is the decrease in RMR not accounted for by changes in fat-free mass and fat mass. ${ }^{29-32}$ In the present investigation, we did not observe adaptive thermogenesis $\sim 12$ hours after the end of the last prolonged walk, maybe due to the vast amount of exercise performed by our subjects, which may result in a prolonged oxygen debt. ${ }^{33}$ Despite the large energy deficit and the reduction in fat mass, SLN expression remained unaltered in the three muscles biopsied, strongly suggesting that, in humans, SLN expression is not downregulated as a mechanism to spare energy. Thus, it is unlikely that SLN plays a role in adaptive thermogenesis in humans. In agreement, genetic deletion of SLN is not associated with reduced resting energy expenditure in mice. ${ }^{34}$

Although the mechanisms that may regulate the levels of SLN expression in skeletal muscle remain unknown, in rats, SLN mRNA increased in the diaphragm after the administration of pharmacological doses of triamcinolone acetate (a potent corticosteroid). ${ }^{35}$ This concurs with the positive association between the changes in SLN protein expression and the changes in cortisol observed in our overweight subjects. Also, in favor of a neuroendocrine (or genetic) regulation of SLN protein expression in skeletal muscle, subjects having low expression levels in the arm muscles had also low expression levels in the vastus lateralis and vice versa.

\section{3 | Sarcolipin protein expression is not altered by short-term low-intensity exercise but it is reduced by repeated sprint exercise}

The present investigation indicates that SLN protein content is not modified by small (trained deltoid in the present study) or very large (vastus lateralis in the present study) amounts of low-intensity exercise. This finding could indicate that to elicit a change in SLN protein expression, a greater exercise intensity may be required.

In contrast with rodent data, ${ }^{3,16}$ here we have demonstrated that a short training program based on repeated high-intensity exercise reduced the SLN protein levels in vastus lateralis despite eliciting much less total energy expenditure than the 4-day walking intervention. A principal difference between prolonged walking and repeated 30 -second all-out sprints is the marked metabolic disturbances evoked by the sprints, which cause substantial lactic acidosis, oxidative stress, and sarcoplasmic reticulum stress. ${ }^{20,36-38}$ Endoplasmic reticulum stress reduces SLN expression in $\mathrm{C} 2 \mathrm{C} 12$ cells. ${ }^{39}$ Genetic ablation of the $\operatorname{Sin}$ gene is associated with higher levels of glycolytic enzymes, including phosphofructokinase 1, hexokinase II, pyruvate kinase muscle, and pyruvate dehydrogenase. $^{40}$ It remains to be determined whether the reduction in SLN protein expression observed in the vastus lateralis may have facilitated the expression of glycolytic enzymes, as reported in previous research. ${ }^{41,42}$

\section{4 | Sarcolipin and performance}

Sarcolipin-overexpressing $\left(\mathrm{SLN}^{\mathrm{OE}}\right)$ mice produce more force and fatigue less than WT. ${ }^{4}$ In the present study, sprint training resulted in improved endurance and enhanced peak 
power and mean power output during the sprints, despite reducing SLN expression in vastus lateralis. Although the training program did not change the mean response of gross efficiency during leg pedaling, there were some individual fluctuations. Interestingly, the change in gross efficiency was inversely associated with the change in SLN. Thus, a reduction of SLN expression could be an adaptive mechanism to spare energy during exercise, likely by increasing the $\mathrm{Ca}^{2+} /$ ATP coupling ratio. Since no significant associations were observed between baseline SLN protein expression levels and performance, nor between changes in SLN and changes in performance with training, our results indicate that SLN is not an important determinant of exercise capacity in humans, apart from a potential small influence on energy efficiency.

\section{5 | Sarcolipin protein expression in human skeletal muscle is not associated with adiposity nor resting energy expenditure in overweight men}

An influence of SLN protein expression in the total fat mass content and RMR has been shown in transgenic mice. ${ }^{5,13}$ Maurya et $\mathrm{al}^{13}$ used both SLN knockout $\left(\mathrm{SLN}^{-/-}\right)$ and skeletal muscle-specific SLN-overexpressing $\left(\mathrm{SLN}^{\mathrm{OE}}\right)$ mice to determine the role of SLN on energy metabolism by pair feeding (fixed calories) and high-fat diet feeding (ad libitum) in mice. Interestingly, these authors observed that when receiving a similar quantity of calories SLN ${ }^{\mathrm{OE}}$ mice lost weight compared with the WT, but $\mathrm{SLN}^{-/-}$mice gained weight. Contrary to this, experiments using human skeletal muscle cells (HSkMC) from lean and obese women, which were differentiated into primary myocytes, reported higher SLN protein expression levels in the myocytes derived from HSkMC of obese lineage. ${ }^{14}$ In the present investigation, despite ample inter-individual differences in the percentage of body fat, no association was observed between the percentage of whole-body fat and SLN protein expression in skeletal muscle. Moreover, after reducing fat mass by $2.8 \mathrm{~kg}$ no significant changes were observed in SLN protein expression in the present investigation. This finding concurs with the lack of association between SLN protein expression and RMR observed here in overweight men. In agreement, Paran et al ${ }^{14}$ have reported lower efficiency of sarcolipin-dependent respiration in myocytes differentiated by the culture of HSkMC obtained humans with severe obesity. According to Paran et al, ${ }^{14}$ this indicates that SLN effects are attenuated in severely obese humans, supporting the idea that SLN does not seem to have an essential role in the regulation of resting metabolic rate in humans.

\section{6 | Limitations}

The mechanisms regulating SLN expression in human skeletal muscle remain unknown. Our subjects were young and middle-aged Caucasians, and it is unknown how race, sex, and age might influence the expression of SLN. The association observed between changes in SLN expression and gross efficiency supports a role for SLN in the regulation of energy expenditure during exercise that should be confirmed with different experimental models. Chronic loading could explain the greater amount of SLN expressed in the vastus lateralis compared to the deltoid muscles, despite the lower amount of fast-twitch fibers in the former. However, studies using strength training programs or analyzing the effects of immobilization are required to confirm this finding. Finally, it is unclear whether an increase of SLN expression could be an effective treatment to counteract obesity in humans without causing muscular dystrophy. ${ }^{43}$

In summary, sarcolipin is expressed in the human skeletal muscle, with the vastus lateralis containing twice as much as the deltoid and approximately $44 \%$ more than the triceps brachii. High-intensity exercise training reduces sarcolipin protein in skeletal muscle, which may explain part of the individual variation in muscle energy efficiency. In contrast to transgenic rodent data, no association between sarcolipin and performance was observed in the present investigation. Sarcolipin protein expression is not associated with adiposity nor resting energy expenditure and is not modified by a severe energy deficit.

\section{5 | PERSPECTIVES}

Overall, these results do not support a role for sarcolipin in the regulation of fat mass in young and middle-aged men. Future research should assess the effects of age, sex, extreme obesity, starvation, immobilization, and mechanical overloading on the level of sarcolipin in human skeletal muscle and associated functional consequences.

\section{ACKNOWLEDGEMENTS}

This study was financed by grants from the Ministerio de Economía y Competitividad (PI14/01509, and FEDER), ACIISI (ProID2017010106), University of Las Palmas de Gran Canaria (ULPGC 2015/05), and Östersund municipality. We offer special thanks to José Navarro de Tuero for his excellent technical assistance. We also would like to express our gratitude to Prof. Robert Boushel for his insightful comments.

\section{CONFLICT OF INTEREST}

All the authors declare that they have no conflict of interest derived from the outcomes of this study. 


\section{AUTHOR CONTRIBUTIONS}

$\mathrm{H}-\mathrm{CH}$, DMA, and JALC conceived and designed the experiment. DMA, MMC, MGR, MMR, H-CH, and JALC collected and analyzed the data. DMA, MMC, MGR, MMR, PPV, $\mathrm{H}-\mathrm{CH}$, and JALC interpreted the data. DMA, MMC, and JALC drafted the manuscript and prepared all figures. All authors read and approved the final version of the manuscript.

\section{DISCLOSURE}

The authors have nothing to disclose.

\section{ORCID}

David Morales-Alamo (iD https://orcid. org/0000-0001-8463-397X

Miriam Gelabert-Rebato (D) https://orcid.

org/0000-0002-8963-5568

Marcos Martin-Rincon (D) https://orcid.

org/0000-0002-3685-2331

Jose A. L. Calbet (DD https://orcid.org/0000-0002-9215-6234

\section{REFERENCES}

1. Babu GJ, Bhupathy P, Carnes CA, Billman GE, Periasamy M. Differential expression of sarcolipin protein during muscle development and cardiac pathophysiology. $\mathrm{J} \mathrm{Mol}$ Cell Cardiol. 2007;43(2):215-222.

2. Bal NC, Maurya SK, Sopariwala DH, et al. Sarcolipin is a newly identified regulator of muscle-based thermogenesis in mammals. Nat Med. 2012;18(10):1575-1579.

3. Riedl I, Osler ME, Bjornholm M, et al. AMPKgamma3 is dispensable for skeletal muscle hypertrophy induced by functional overload. Am J Physiol Endocrinol Metab. 2016;310(6):E461-472.

4. Sopariwala DH, Pant M, Shaikh SA, et al. Sarcolipin overexpression improves muscle energetics and reduces fatigue. $J$ Appl Physiol (1985). 2015;118(8):1050-1058.

5. Maurya SK, Periasamy M. Sarcolipin is a novel regulator of muscle metabolism and obesity. Pharmacol Res. 2015;102:270-275.

6. Campbell KL, Dicke AA. Sarcolipin makes heat, but is it adaptive thermogenesis? Front Physiol. 2018;9:714.

7. Muller MJ, Bosy-Westphal A, Kutzner D, Heller M. Metabolically active components of fat-free mass and resting energy expenditure in humans: recent lessons from imaging technologies. Obes Rev. 2002;3(2):113-122.

8. Periasamy M, Maurya SK, Sahoo SK, et al. Role of SERCA pump in muscle thermogenesis and metabolism. Compr Physiol. 2017;7(3):879-890.

9. Smith IC, Bombardier E, Vigna C, Tupling AR. ATP consumption by sarcoplasmic reticulum $\mathrm{Ca}(2)(+)$ pumps accounts for $40-50 \%$ of resting metabolic rate in mouse fast and slow twitch skeletal muscle. PLoS ONE. 2013;8(7):e68924.

10. de Meis L. Uncoupled ATPase activity and heat production by the sarcoplasmic reticulum $\mathrm{Ca}^{2+}$-ATPase. Regulation by ADP. J Biol Chem. 2001;276(27):25078-25087.

11. Smith WS, Broadbridge R, East JM, Lee AG. Sarcolipin uncouples hydrolysis of ATP from accumulation of $\mathrm{Ca}^{2+}$ by the $\mathrm{Ca}^{2+}$ ATPase of skeletal-muscle sarcoplasmic reticulum. Biochem J. 2002;361(Pt 2):277-286.
12. Fajardo VA, Bombardier E, Vigna $C$, et al. Co-expression of SERCA isoforms, phospholamban and sarcolipin in human skeletal muscle fibers. PLoS ONE. 2013;8(12):e84304.

13. Maurya SK, Bal NC, Sopariwala DH, et al. Sarcolipin Is a key determinant of the basal metabolic rate, and its overexpression enhances energy expenditure and resistance against diet-induced obesity. J Biol Chem. 2015;290(17):10840-10849.

14. Paran CW, Verkerke AR, Heden TD, et al. Reduced efficiency of sarcolipin-dependent respiration in myocytes from humans with severe obesity. Obesity. 2015;23(7):1440-1449.

15. Odermatt A, Taschner PE, Scherer SW, et al. Characterization of the gene encoding human sarcolipin (SLN), a proteolipid associated with SERCA1: absence of structural mutations in five patients with Brody disease. Genomics. 1997;45(3):541-553.

16. Fajardo VA, Rietze BA, Chambers PJ, et al. Effects of sarcolipin deletion on skeletal muscle adaptive responses to functional overload and unload. Am J Physiol Cell Physiol. 2017;313(2):C154 $-\mathrm{C} 161$.

17. Calbet JAL, Ponce-Gonzalez JG, Calle-Herrero J, et al. Exercise preserves lean mass and performance during severe energy deficit: the role of exercise volume and dietary protein content. Front Physiol. 2017;8:483.

18. Calbet JA, Ponce-Gonzalez JG, Perez-Suarez I, de la Calle HJ, Holmberg HC. A time-efficient reduction of fat mass in 4 days with exercise and caloric restriction. Scand J Med Sci Sports. 2015;25(2):223-233.

19. Zinner C, Morales-Alamo D, Ortenblad N, et al. The physiological mechanisms of performance enhancement with sprint interval training differ between the upper and lower extremities in humans. Front Physiol. 2016;7:426.

20. Larsen FJ, Schiffer TA, Ortenblad N, et al. High-intensity sprint training inhibits mitochondrial respiration through aconitase inactivation. FASEB J. 2016;30(1):417-427.

21. Perez-Suarez I, Ponce-Gonzalez JG, de La Calle-Herrero J, et al. Severe energy deficit upregulates leptin receptors, leptin signaling, and PTP1B in human skeletal muscle. J Appl Physiol. 2017;123(5):1276-1287.

22. Calbet JA, Dorado C, Diaz-Herrera P, Rodriguez-Rodriguez LP. High femoral bone mineral content and density in male football (soccer) players. Med Sci Sports Exerc. 2001;33(10):1682-1687.

23. Peronnet F, Massicotte D. Table of nonprotein respiratory quotient: an update. Can J Sport Sci. 1991;16(1):23-29.

24. Martin-Rincon M, Gonzalez-Henriquez JJ, Losa-Reyna J, et al. Impact of data averaging strategies on $\mathrm{VO}_{2} \max$ assessment: mathematical modelling and reliability. Scand J Med Sci Sports. 2019;29(10):1473-1488.

25. Bass JJ, Wilkinson DJ, Rankin D, et al. An overview of technical considerations for Western blotting applications to physiological research. Scand J Med Sci Sports. 2017;27(1):4-25.

26. Yonan CR, Duong PT, Chang FN. High-efficiency staining of proteins on different blot membranes. Anal Biochem. 2005;338(1):159-161.

27. Guadalupe-Grau A, Rodriguez-Garcia L, Torres-Peralta R, et al. Greater basal skeletal muscle AMPKalpha phosphorylation in men than in women: associations with anaerobic performance. Eur $J$ Sport Sci. 2016;16(4):455-464.

28. Martin-Rincon M, Perez-Suarez I, Perez-Lopez A, et al. Protein synthesis signaling in skeletal muscle is refractory to whey protein ingestion during a severe energy deficit evoked by 
prolonged exercise and caloric restriction. Int $J$ Obes (Lond). 2019;43(4):872-882.

29. Leibel RL, Rosenbaum M, Hirsch J. Changes in energy expenditure resulting from altered body weight. $N$ Engl $\mathrm{J}$ Med. 1995;332(10):621-628.

30. Muller MJ, Enderle J, Pourhassan M, et al. Metabolic adaptation to caloric restriction and subsequent refeeding: the Minnesota Starvation Experiment revisited. Am J Clin Nutr. 2015;102(4):807-819.

31. Schwartz A, Doucet E. Relative changes in resting energy expenditure during weight loss: a systematic review. Obes Rev. 2010;11(7):531-547.

32. Johannsen DL, Knuth ND, Huizenga R, Rood JC, Ravussin E, Hall KD. Metabolic slowing with massive weight loss despite preservation of fat-free mass. J Clin Endocrinol Metab. 2012;97(7):2489-2496.

33. Maehlum S, Grandmontagne M, Newsholme EA, Sejersted OM. Magnitude and duration of excess postexercise oxygen consumption in healthy young subjects. Metabolism. 1986;35(5):425-429.

34. Bombardier E, Smith IC, Vigna C, Fajardo VA, Tupling AR. Ablation of sarcolipin decreases the energy requirements for $\mathrm{Ca} 2+$ transport by sarco(endo)plasmic reticulum $\mathrm{Ca}^{2+}$-ATPases in resting skeletal muscle. FEBS Lett. 2013;587(11):1687-1692.

35. Gayan-Ramirez G, Vanzeir L, Wuytack F, Decramer M. Corticosteroids decrease mRNA levels of SERCA pumps, whereas they increase sarcolipin mRNA in the rat diaphragm. $J$ Physiol. 2000;524(Pt 2):387-397.

36. Morales-Alamo D, Calbet JA. Free radicals and sprint exercise in humans. Free Radic Res. 2014;48(1):30-42.

37. Morales-Alamo D, Ponce-Gonzalez JG, Guadalupe-Grau A, et al. Increased oxidative stress and anaerobic energy release, but blunted Thr172-AMPKalpha phosphorylation, in response to sprint exercise in severe acute hypoxia in humans. J Appl Physiol. 2012;113(6):917-928.

38. Bohnert KR, McMillan JD, Kumar A. Emerging roles of ER stress and unfolded protein response pathways in skeletal muscle health and disease. J Cell Physiol. 2018;233(1):67-78.

39. Takahashi N, Kimura AP, Naito S, et al. Sarcolipin expression is repressed by endoplasmic reticulum stress in $\mathrm{C} 2 \mathrm{C} 12$ myotubes. J Physiol Biochem. 2017;73(4):531-538.

40. Maurya SK, Herrera JL, Sahoo SK, et al. Sarcolipin signaling promotes mitochondrial biogenesis and oxidative metabolism in skeletal muscle. Cell Rep. 2018;24(11):2919-2931.

41. Linossier MT, Dormois D, Perier C, Frey J, Geyssant A, Denis C. Enzyme adaptations of human skeletal muscle during bicycle short-sprint training and detraining. Acta Physiol Scand. 1997;161(4):439-445.

42. Rodas G, Ventura JL, Cadefau JA, Cusso R, Parra J. A short training programme for the rapid improvement of both aerobic and anaerobic metabolism. Eur J Appl Physiol. 2000;82(5-6):480-486.

43. Voit A, Patel V, Pachon R, et al. Reducing sarcolipin expression mitigates Duchenne muscular dystrophy and associated cardiomyopathy in mice. Nat Commun. 2017;8(1):1068.

How to cite this article: Morales-Alamo D, MartinezCanton M, Gelabert-Rebato M, et al. Sarcolipin expression in human skeletal muscle: Influence of energy balance and exercise. Scand J Med Sci Sports. 2020;30:408-420. https://doi.org/10.1111/sms.13594 